\title{
Numerical and perturbative analysis on non-axisymmetric Homann stagnation-point flow of Maxwell fluid
}

\author{
M. Sajid' 1 (D. M. Jagwal ${ }^{2} \cdot$ I. Ahmad ${ }^{2}$
}

Received: 12 August 2020 / Accepted: 4 February 2021 / Published online: 8 March 2021

(c) The Author(s) 2021 OPEN

\begin{abstract}
In this paper, we examined the numerical and perturbative analysis of non-Newtonian fluid towards non-axisymmetric Homann stagnation-point flow. The Maxwell fluid model is applied to investigate the behavior of viscoelastic fluid for this particular geometry. The influence of Maxwell parameter $\beta_{1}$ and ratio $\gamma$ on different profiles are addressed in this analysis. The governed partial differential equations are reduced to ordinary differential equations with the help of similarity transformations. The numerical and perturbative outcomes of the resulting system of differential equations are obtained by applying the shooting technique. The solution is achieved for diverse values of relaxation time parameter $\beta_{1}$ and ratio $\gamma$. The wall shear stress is compared to their large- $\gamma$ asymptotic behaviors and displacement thicknesses are also presented. The numerical data for velocity profiles are obtained in terms of plots. It is predicted through analysis that a gradual increase in relaxation time raises wall skin friction components. On the other hand, velocity decreases which constitutes to reduce the reverse flow. Meanwhile, displacement thicknesses in $x$ and $y$ direction decreases. However, three-dimensional displacement thickness increases due to more viscoelastic material like Maxwell fluid than viscous fluid.
\end{abstract}

Keywords Non-axisymmetric flow · Maxwell fluid · Homann flow · Stagnation-point flow $\cdot$ Shooting method

\section{List of symbols}

$\checkmark \quad$ Fluid velocity

$\mathrm{u}, \mathrm{v}, \mathrm{w}$ Velocity components

$x, y, z \quad$ Space coordinates

a Strain rate

b Shear rates

$Y \quad$ Shear to strain rate ratio

$\varepsilon \quad$ Small parameter in perturbation

$\eta \quad$ Similarity variable

$\xi \quad$ New variabler

$\lambda \quad$ Maxwell prameter

$\beta_{1} \quad$ Deborah number

$\mu \quad$ Liquid dynamic viscosity

$v \quad$ Kinematic viscosity of the liquid

$\rho \quad$ Density of the liquid

$\tau \quad$ Stress tensor
S Extra stress tensor for Maxwell fluid

$A_{1} \quad$ First Rivlin-Ericksen tensor

$\mathrm{p} \quad$ Pressure

$\mathrm{p}_{0} \quad$ Stagnation pressure

$\delta \quad$ Boundary-layer thickness

a Boundary-layer thickness in $\mathrm{x}$ - direction

$\beta \quad$ Boundary-layer thickness in $y$ - direction

\section{Introduction}

Non-Newtonian fluids exhibit non-linear deformation during flow because of their complex structural composition. The mathematical modeling of such fluid displays a nonlinear differential system which is a challenge for scientists in the recent area. These fluids are abundantly found

M. R. Jagwal, mrafi.math@ajku.edu.pk | 'Department of Mathematics and Statistics, International Islamic University, Islamabad 44000, Pakistan. ${ }^{2}$ Department of Mathematics, University of Azad Jammu and Kashmir, Muzaffarabad 13100, Pakistan. 
naturally in various applications including pharmaceutical, plastic, and petrochemical industries. These fluids having particles of large-chain biopolymers, hydrogels, and emulsions are found to be soft and complex material. On the other hand, many complex fluids behave exotically (shearthickening) when shear stress is applied and some of them exhibit both elastic and viscous effects. This is because of dissipative and elastic mechanisms. The rheological investigations on such fluids are made to predict the behavior of these fluids against shear stress. The Maxwell fluid model is constructed by using Hookean spring combined with a dashpot intended to mechanically describe viscoelastic materials. The Maxwell fluid model was firstly established by Maxwell [1] to study the visco-elastic behavior of air. The combination of basic elements in different arrangements can be made to reproduce more complex viscoelastic behavior. The important characteristics of Maxwell fluid are to exhibit stress relaxation (memory effects). The literature reveals that many attempts were made to investigate the rheological effects of a Maxwell fluid for different geometries in hydrodynamic boundary-layer. The viscoelastic Upper convective Maxwell fluid properties were firstly discussed by Olsson and Yström [2]. The Maxwell fluid flow near stagnation-point using spectral collocationpoint method was examined by Sadeghy et al. [3]. In the article, they conclude a precise result for the second grade upper convective Maxwell fluid model. Kumari and Nath [4] investigated the effects of buoyancy force on stagnation-point flow of Maxwell fluid. The obtained results indicate that the increasing viscoelasticity implies a reduction in fluid velocity. The flow of electrically conducted Maxwell fluid near stagnation-point over continuously deforming sheet was analyzed by Hayat et al. [5]. Mukhopadhyay [6] showed the heat transfer in unsteady Maxwell fluid on the stretching sheet. Motsa et al. [7] applied successive linearization to obtain the result for Maxwell fluid flow over a shrinking surface. Some of the important characteristics of Maxwell boundary layer fluid flow were analyzed by Renardy and Wang [8]. Bhattacharyya et al. [9] explored multiple solutions of Maxwell fluid flow over a shrinking porous surface. Stagnation-point flow of Jeffery nanofluid with heat source towards stretching sheet was numerically studied by Ramesh et al. [10]. Further, Ramesh et al. [11] discussed stagnation-point flow of Maxwell nanofluid flow towards the porous surface. They analyzed the heating mechanism using nanoparticles in Maxwell fluid. Prasannakumara et al. [12] considered stagnation-point flow of Jeffery nanofluid flow towards stretching surface with melting and radiation effects. Mushtaq et al. [13] explained the buoyancy effects near the stagnation-point flow of Maxwell fluid with non-Fourier heat flux. Some recent investigations on this direction are found in the literature some of them are included in ref. [14, 15].
Axisymmetric stagnation-point fluid flows have many fundamental properties in fluid dynamics due to their industrial application. Hiemenz [16] was the first to solve the problem for planner stagnation-point flow. Later on, the axisymmetric stagnation-point flows were discussed by Homann [17]. The problem in which two orthogonal Hiemenz flows are discussed were examined by Howarth [18]. In his article, Howarth explains the solution in the range $c \in[0,1]$ and suggested that the solution can be extended to outside the interval using suitable transformation. Working in this direction, Davey [19] presented disagreement with Howarth's paper. He pointed out that the solution obtained for $c \in[-1,0]$ could not be achieved from those created for $c \in[0,1](c=b / a$, where $a$ and $b$ represents the rate of strain). Davey finds out that this is the saddle-point solution of Howarth's equations. The three-dimensional fluid flow nearby the two-dimensional stagnation-point is described by Davey and Schofield [20]. Following the same direction, the dual solution for Howarth's equations was reported by Schofield and Davey [21]. Sajid et al. [22] discussed Homann's flow of a micropolar fluid near a spiraling disk.

Non-axisymmetric stagnation-point flows are involved in many manufacturing procedures such as textile technology, printing industry, water-show technology, cooling and centrifugal processes in the industry, and calcinations of cement. Due to its abundant industrial importance, in the last decade, different attempts were made on nonaxisymmetric Homann stagnation-point flow. The viscous Homann non-axisymmetric stagnation-point flow was presented by Weidman [23]. He explains how periodic term affects the stagnation-point of Homann flow. The heat transfer analysis on non-axisymmetric Homann stagnation-point flow over a stretching surface was investigated by Mahapatra and Sidui [24]. The analysis of heat transfer in the unsteady case on Homann nonaxisymmetric stagnation-point flow over a rigid surface was made by Mahapatra and Sidui [25]. The asymptotic and numerical explanation of non-axisymmetric MHD boundary-layer stagnation-point flow was obtained by Kudenatti and Kirsur [26]. They found out that viscous fluid flow is due to the applied magnetic field and the effects of the outer stream. Homann non-axisymmetric stagnation-point flow for mixed convection over a vertical flat plate in an incompressible viscous fluid was considered by Lok et al. [27]. Mahapatra and Sidui [28] investigated the study of non-axisymmetric stagnation-point flow of viscoelastic Walter-B fluid toward a rigid surface. Ahmad et al. [29] explored the study of non-axisymmetric Homann stagnation-point for nanofluid. They applied the shooting method for both asymptotic and numerical results. Zainal et al. [30] examined stability analysis for MHD threedimensional unsteady flow of hybrid nanofluid towards 
non-axisymmetric Homann stagnation-point. Recently, Jagwal et al. [31] find out numerical and asymptotic solution for non-axisymmetric Homann stagnation-point of Maxwell nanofluid toward fixed plate.

Due to abundant application in industries, the author's interest is to explore the study of Maxwell fluid towards non-axisymmetric stagnation point. The problem is modeled in the Cartesian coordinate. To the best of the author's knowledge, no one had considered the Homann stagnation-point flow in the Maxwell fluid till now. The results are interesting, and the present work will be a useful contribution to the scope of non-axisymmetric Homann stagnation-point flow in the Maxwell fluid. The effects of involved parameters are analyzed on velocities, wall shear, and displacement thicknesses. The asymptotic solution for large $-\gamma$ is also presented in the paper.

The paper arrangement is as follows. The problem formulation is described in Sect. 2 whereas Sect. 3 includes asymptotic behavior for large $-\gamma$ of the problem. Sect. 4 deals with numerical results and Sect. 5 concludes the paper.

\section{Problem formulation}

Consider the steady three-dimensional incompressible flow of Maxwell fluid is impinging toward stationary surface at rest. Furthermore, the flow region is $z>0$ and the stagnation-point coincides with plane $z=0$ as shown in Fig. 1.

The constitutive equation of Maxwell fluid (Olsson et al. [2]) are:

$$
\tau_{i j}=-P I+S,
$$

in the Eq. (1), $S$ satisfies the following relation

$$
S+\lambda \frac{D S}{D t}=\mu A_{1} \text {. }
$$

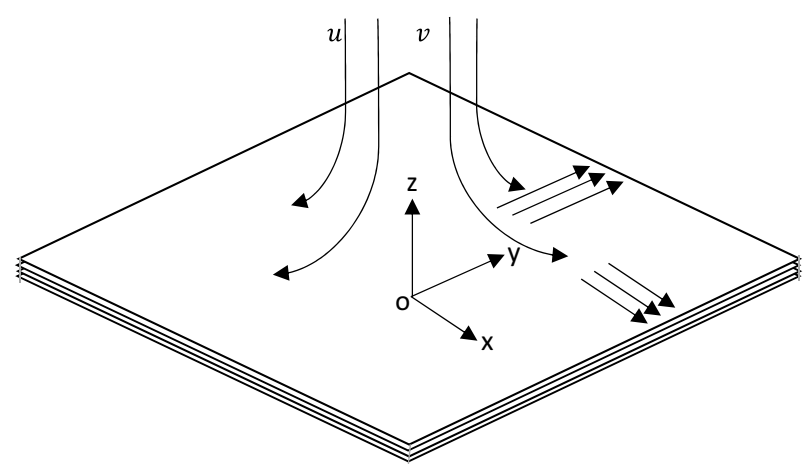

Here, $\lambda$ is time relaxation parameter, $A_{1}$ is first RivlinEricksen tensor, $\mu$ is the dynamic viscosity and $\frac{D S}{D t}$ upper convective time derivative. Consider fluid is impinged vertically toward surface which is at rest and flows radially in all the direction near stagnation-point. The momentum equations along with continuity equation are

$\frac{\partial u}{\partial x}+\frac{\partial v}{\partial y}+\frac{\partial w}{\partial z}=0$,

$u \frac{\partial u}{\partial x}+v \frac{\partial u}{\partial y}+w \frac{\partial u}{\partial z}=\frac{1}{\rho}\left(\frac{\partial \tau_{11}}{\partial x}+\frac{\partial \tau_{12}}{\partial y}+\frac{\partial \tau_{13}}{\partial z}\right)$

$u \frac{\partial v}{\partial x}+v \frac{\partial v}{\partial y}+w \frac{\partial v}{\partial z}=\frac{1}{\rho}\left(\frac{\partial \tau_{21}}{\partial x}+\frac{\partial \tau_{22}}{\partial y}+\frac{\partial \tau_{23}}{\partial z}\right)$

$u \frac{\partial w}{\partial x}+v \frac{\partial w}{\partial y}+w \frac{\partial w}{\partial z}=\frac{1}{\rho}\left(\frac{\partial \tau_{31}}{\partial x}+\frac{\partial \tau_{32}}{\partial y}+\frac{\partial \tau_{33}}{\partial z}\right)$,

where $u, v$ and $w$ are the velocities of fluid in $x, y$ and $z$ directions.

The boundary conditions and similarity variables are defined as in [23],

$$
\begin{aligned}
& u=0, v=0, w=0, a t z=0, \\
& u=(a+b) x, v=(a-b) y, w=-2 a z, a t z \rightarrow \infty .
\end{aligned}
$$

$$
\begin{aligned}
u(x, y, z) & =(a+b) x f^{\prime}(\eta), v(x, y, z)=(a-b) y g^{\prime}(\eta), w(z) \\
& =-\sqrt{\frac{v}{a}}[(a+b) f(\eta)+(a-b) g(\eta)], \eta=\sqrt{\frac{a}{v} z .}
\end{aligned}
$$

Where, $b$ is rate of shear and $a$ is rate of strain. Applying boundary layer approximation and then using transformations (9) to Eqs. $(4,5,6)$, one gets

$$
\begin{aligned}
& f^{\prime \prime \prime}-(1+\gamma)\left(f^{\prime 2}-f f^{\prime \prime}-1\right)+\beta_{1}(1+\gamma)^{2}\left(2 f f^{\prime} f^{\prime \prime \prime}-f^{2} f^{\prime \prime}\right) \\
& \quad+2 \beta_{1}\left(1-\gamma^{2}\right)\left(g f^{\prime} f^{\prime \prime}-f g f^{\prime \prime \prime}\right)-\beta_{1}(1-\gamma)^{2} g^{2} f^{\prime \prime \prime}+(1-\gamma) g f^{\prime \prime}=0, \\
& g^{\prime \prime \prime}-(1-\gamma)\left(g^{\prime 2}-g g^{\prime \prime}-1\right)+\beta_{1}(1-\gamma)^{2}\left(2 g g^{\prime} g^{\prime \prime \prime}-g^{2} g^{\prime \prime}\right) \\
& \quad+2 \beta_{1}\left(1-\gamma^{2}\right)\left(f g^{\prime} g^{\prime \prime}-g f g^{\prime \prime \prime}\right)-\beta_{1}(1+\gamma)^{2} f^{2} g^{\prime \prime \prime}+(1+\gamma) f g^{\prime \prime}=0,
\end{aligned}
$$

where, $\gamma=\frac{b}{a}$ is ratio of shear to strain rate, $\beta_{1}=\lambda a$ is Maxwell parameter, the restrictions on the boundaries are

$$
f(0)=0, f^{\prime}(0)=0, g(0)=0, g^{\prime}(0)=0, f^{\prime}(\infty)=1, g^{\prime}(\infty)=1
$$

Inspection of (10) reveals the reflexive symmetries

Fig. 1 Physical representations of the problem 
$f(\eta,-\gamma)=g(\eta, \gamma), g(\eta,-\gamma)=f(\eta, \gamma)$,

The pressure field for in this case is

$$
\begin{aligned}
p= & p_{0}-\rho\left[\frac{(a+b)^{2}}{2} x^{2}+\frac{(a-b)^{2}}{2} y^{2}+a v\left\{\frac{[(1+\gamma) f+(1-\gamma) g]^{2}}{2}+\left[(1+\gamma) f^{\prime}+(1-\gamma) g^{\prime}\right]-\lambda a[(1+\gamma) f+(1-\gamma) g]^{2}\left[(1+\gamma) f^{\prime}+(1-\gamma) g^{\prime}\right]\right\}\right] \\
& +2 \rho \lambda \int w(z)\left(\frac{\partial w}{\partial z}\right)^{2} d z .
\end{aligned}
$$

where $\epsilon=\gamma^{-1 / 2} \ll 1$. Therefore the flow equations become
Here, $p_{0}$ is a stagnation pressure.

The boundary-layer displacement thickness $\delta_{x}$ and $\delta_{y}$ can be obtained from the relations below

$\sqrt{\frac{a}{v}} \delta_{x}=\int_{0}^{\infty}\left[1-f^{\prime}(\eta)\right] d \eta \equiv \alpha, \sqrt{\frac{a}{v}} \delta_{y}=\int_{0}^{\infty}\left[1-g^{\prime}(\eta)\right] d \eta \equiv \beta$

The relation for three-dimensional boundary layer thickness as $\eta \rightarrow 0$ are obtained using transformations $F(\eta)=1-f^{\prime}(\eta)$ and $G(\eta)=1-g^{\prime}(\eta)$ to linearized Eq. (10) are.

$\delta_{1 x}=\frac{\left[(1+\gamma)+2 \beta_{1}(1+\gamma)^{2}\right] \alpha+\left[(1-\gamma)+2 \beta_{1}\left(1-\gamma^{2}\right)\right] \beta}{2\left[1+2 \beta_{1}(1+\gamma)\right]}$,

$\delta_{1 y}=\frac{\left[(1+\gamma)+2 \beta_{1}\left(1-\gamma^{2}\right)\right] \alpha+\left[(1-\gamma)+2 \beta_{1}(1-\gamma)^{2}\right] \beta}{2\left[1+2 \beta_{1}(1-\gamma)\right]}$.

Taking mean to developed a single relation for threedimensional boundary layer thickness that preserve the overall contribution of the above relation is

$\delta_{1}=\frac{\delta_{1 x}+\delta_{1 y}}{2}$

where, $\delta_{1 x}$ and $\delta_{1 y}$ in $x$ and $y$-direction respectively (Davey [19]).

The shear stress at the wall can be calculated from the relation

$\tau=\mu\left[\tau_{13} i+\tau_{23} j\right]_{z=0}=\frac{\mu a^{3 / 2}}{\nu^{1 / 2}}\left[(1+\gamma) f^{\prime \prime}(0) x i+(1-\gamma) g^{\prime \prime}(0) y j\right]$,

where, $i$ and $j$ are the unit vectors in $x$ and $y$-direction respectively.

\section{Large $-\gamma$ asymptotic behavior}

The solutions for asymptotic behavior using $\gamma \gg 1$, can be achieved by transforming given differential equation with the help of transformation given

$f(\eta)=\epsilon F(\xi), g(\eta)=\epsilon G(\xi), \xi=\frac{\eta}{\epsilon}, \beta_{1}=\epsilon^{2}$,

$$
\begin{aligned}
& F^{\prime \prime \prime}-\left(\varepsilon^{2}+1\right)\left[F^{2}-F F^{\prime \prime}-1\right]+\left(\varepsilon^{2}+1\right)^{2}\left[2 F F^{\prime} F^{\prime \prime}-F^{2} F^{\prime \prime \prime}\right] \\
& \quad+2\left(\varepsilon^{4}-1\right)\left[G F^{\prime} F^{\prime \prime}-F G F^{\prime \prime \prime}\right]-\left(\varepsilon^{2}-1\right)^{2} G^{2} F^{\prime \prime \prime}+\left(\varepsilon^{2}-1\right) G F^{\prime \prime}=0,
\end{aligned}
$$

$$
\begin{aligned}
& G^{\prime \prime \prime}-\left(\varepsilon^{2}-1\right)\left[G^{\prime 2}-G G^{\prime \prime}-1\right]+\left(\varepsilon^{2}-1\right)^{2}\left[2 G G^{\prime} G^{\prime \prime}-G^{2} G^{\prime \prime \prime}\right] \\
& -2\left(\varepsilon^{4}-1\right)\left[F G^{\prime} G^{\prime \prime}-G F G^{\prime \prime \prime}\right]-\left(\varepsilon^{2}+1\right)^{2} F^{2} G^{\prime \prime \prime}+\left(\varepsilon^{2}+1\right) F G^{\prime \prime}=0,
\end{aligned}
$$

where, prime denotes the derivatives of functions with respect to $\xi$. Applying regular perturbation expansions.

$F(\xi)=F_{0}(\xi)+\epsilon^{2} F_{1}(\xi)+\ldots, G(\xi)=G_{0}(\xi)+\epsilon^{2} G_{1}(\xi)+\ldots$,

a sequence of boundary value problem at each $\epsilon^{2 n}$ can be obtained, here we take only $\epsilon^{0}$ order terms

$$
\begin{aligned}
& F_{0}^{\prime \prime \prime}+F_{0} F^{\prime \prime}-F_{0}^{\prime 2}+1+2 F_{0} F_{0}^{\prime} F_{0}^{\prime \prime}-F_{0}^{\prime 2} F_{0}^{\prime \prime \prime} \\
& \quad-2 G_{0} F_{0}^{\prime} F_{0}^{\prime \prime}+2 F_{0} G_{0} F_{0}^{\prime \prime \prime}-G_{0}^{\prime 2} F_{0}^{\prime \prime \prime}-G_{0} F_{0}^{\prime \prime}=0,
\end{aligned}
$$

$$
\begin{aligned}
& G_{0}^{\prime \prime \prime}-G_{0} G^{\prime \prime}+G_{0}^{\prime 2}-1+2 G_{0} G_{0}^{\prime} G_{0}^{\prime \prime}-G_{0}^{\prime 2} G_{0}^{\prime \prime \prime} \\
& \quad-2 F_{0} G_{0}^{\prime} G_{0}^{\prime \prime}+2 G_{0} F_{0} G_{0}^{\prime \prime \prime}-F_{0}^{\prime 2} G_{0}^{\prime \prime \prime}+F_{0} G_{0}^{\prime \prime}=0 .
\end{aligned}
$$

The boundary conditions are

$F_{0}(0)=0, G_{0}(0)=0, F_{0}^{\prime}(0)=0, G_{0}^{\prime}(0)=0, G_{0}^{\prime}(\infty)=1, F_{0}^{\prime}(\infty)=1$.

The displacement thicknesses

$\int_{0}^{\infty}\left[1-F_{0}^{\prime}(\xi)\right] d \xi=\alpha_{0}=0.610, \int_{0}^{\infty}\left[1-G_{0}^{\prime}(\xi)\right] d \xi=\beta_{0}=2.971$.

The shear stress parameters at the wall for large $-\gamma$ are

$f^{\prime \prime}(0) \sim \gamma^{1 / 2} F_{0}^{\prime \prime}(0)=1.3772 \gamma^{1 / 2}, g^{\prime \prime}$

(0) $\sim 4 \gamma^{1 / 2} G_{0}^{\prime \prime}(0)=-0.6888 \gamma^{1 / 2}$.

The displacement thickness takes the form

$\alpha \sim \gamma^{-1 / 2} \alpha_{0}, \beta \sim \gamma^{-1 / 2} \beta_{0}, \delta_{1} \sim \frac{1}{2 \gamma^{1 / 2}}\left[\left.\delta_{1 x}\right|_{\alpha_{0}}+\left.\delta_{1 y}\right|_{\beta_{0}}\right]$.

From the above equations it is clear that shear stress parameters raise infinity and the displacement thickness $\delta_{x}$ and $\delta_{y}$ tends to zero as $\gamma \rightarrow \infty$. The prominent behavior for 
$\delta_{1} \sim-\frac{1}{2}\left[\left(\beta_{0}-\alpha_{0}\right)+4 \beta_{1} \beta_{0} \gamma\right]\left(1-2 \beta_{1}\right) \gamma^{1 / 2}$

as $\gamma \rightarrow \infty$ move toward negative infinity. It is interesting to note that for $\beta_{1}=0$ the relation for viscous fluid can be achieved.

\section{Numerical result and discussion}

To solve the highly non-linear system (10) subjected to (11) numerically, we have applied the shooting method. The Newton iterative processes are applied to ensure quadratic convergence. The numerical results are achieved for different values of Maxwell parameter $\beta_{1}$ and $\gamma$. The effects of $\beta_{1}$ on velocity, shear stress parameters and displacement thicknesses are also investigated. For $\beta_{1}=0$, our problem reduces to Weidman [23] problem for a viscous fluid and for $\gamma=0$, axisymmetric flow situation are achieved.

In Table 1, the comparison of the numerical and perturbative solution of shear stresses are analyzed for selected values of $\gamma$ and $\beta_{1}$. It can be seen from numerical values, the shear stress $f^{\prime \prime}(0)$ increases as relaxation time $\beta_{1}$ is increased while the opposite trend in $g^{\prime \prime}(0)$ is analyzed when $\gamma$ varies from 0 to 4 . The asymptotic solution for $f^{\prime \prime}(0)$ increases and moves much closer to the numerical solution as $\gamma$ moves from 0 to 4 . On the other hand, asymptotic solution for $g^{\prime \prime}(0)$ gets increased in this interval but remains little away from numeric values. The complete analysis over $-10 \leq \gamma \leq 10$ for different value of $\beta_{1}$ are ploted in Fig. 2 .

Figure 2 illustrates the behavior of shear stresses at the wall $f^{\prime \prime}(0)$ and $g^{\prime \prime}(0)$ which are ranging from $-10 \leq \gamma \leq 10$ for $\beta_{1}=0.0, \beta_{1}=0.01$ and $\beta_{1}=0.02$. The fundamental numerical outcomes are plotted below where solid lines represent numeric values of shear stress parameters while
Table 1 Comparison of numerical and first order perturbation analysis of shear stresses

\begin{tabular}{lllllll}
\hline$\beta_{1}$ & $\gamma$ & $f^{\prime \prime}(0)$ & $g^{\prime \prime}(0)$ & $\gamma$ & $\gamma^{\frac{1}{2}} F_{0}^{\prime \prime}(0)$ & $\gamma^{\frac{1}{2}} G_{0}^{\prime \prime}(0)$ \\
\hline 0 & 0 & 1.479636 & 1.479636 & 0 & 0 & 0 \\
& 2 & 2.150958 & 0.751053 & 2 & 1.947774 & -0.243568 \\
& 4 & 2.742920 & 0.039788 & 4 & 2.754569 & -0.344457 \\
0.01 & 0 & 1.481195 & 1.481195 & & & \\
& 2 & 2.155826 & 0.750030 & & & \\
& 4 & 2.757052 & 0.030769 & & & \\
& 0.02 & 0 & 1.482768 & 1.482768 & & \\
& 2 & 2.160772 & 0.748999 & & & \\
& 4 & 2.771752 & 0.021368 & & & \\
\hline
\end{tabular}

Fig. 2 Variation in $f^{\prime \prime}(0)$ and $g^{\prime \prime}(0)$ along $\gamma$ when $\beta_{1}=0.0,0.01,0.02$. Solid lines represent numerical solutions whereas dotted lines indicate asymptotic behaviors for large- $\gamma$

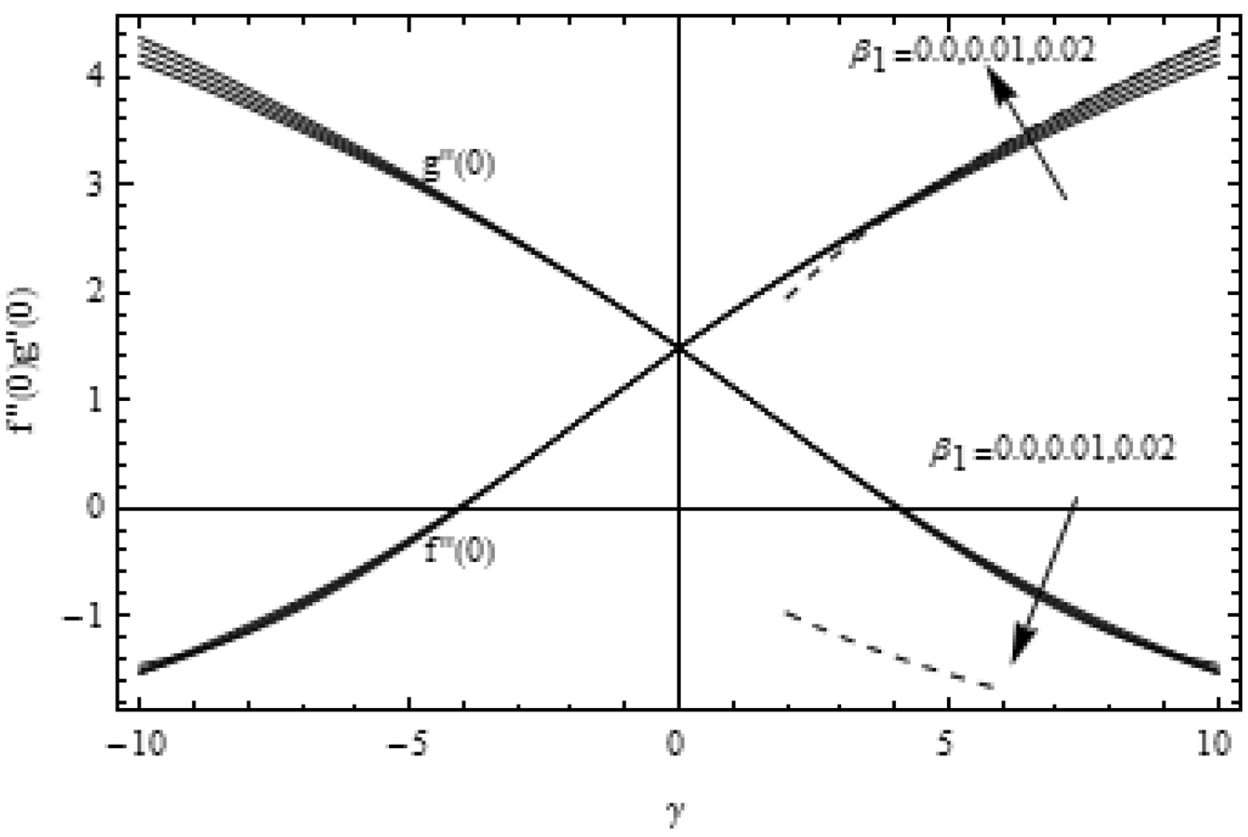

SN Applied Sciences 
dotted lines exhibit asymptotic behavior for large- $\gamma$. In these numerical and perturbative results, it is clear that when $\gamma$ is increased from 0 to 10 , the shear stress $f^{\prime \prime}(0)$ gradually increases whereas $g^{\prime \prime}(0)$ reduces in this particular range of $\gamma$. On the other hand, when $\gamma$ varies from -10 to 0 opposite behavior can be seen, $g^{\prime \prime}(0)$ rises whereas $f^{\prime \prime}(0)$ decreases. In the whole interval $-10 \leq \gamma \leq 10$, the shear stress at the wall exhibits reflection symmetry about $\gamma=0$ which is expressed in (12). It is interesting to note that for $\beta_{1}=0$ (there is no contribution of viscoelastic effects), the results for viscous flow (Weidman [23]) are recovered. It can be seen that for $\beta_{1}=0.01$ and $\beta_{1}=0.02$ as $\gamma \rightarrow \pm \infty$, the wall shear stress parameters $f^{\prime \prime}(0)$ and $g^{\prime \prime}(0)$ grow rapidly in upward direction above the horizontal axis compared with a viscous fluid. This implies that Maxwell fluid requires more shear to deform due to viscoelastic behavior than viscous fluid. In the upper half of the Fig. 2, the asymptotic solution of $g^{\prime \prime}(0)$ best agrees with numerical solution. This asymptotic solutions remain the same for $\gamma \geq 4$ when $\beta_{1}=0.02$. The wall shear stress raises much significantly away from the axisymmetric case $(\gamma=0)$. In lower half of the Fig. 2 , it can be observed that the shear stresses decrease as relaxation time parameter $\beta_{1}$ increases. It can be seen from the plot that both shear stress parameters behave the same in the interval $-2.7 \leq \gamma \leq 2.7$. Near axisymmetric case $(\gamma=0)$, the curves overlap which show that variation in relaxation time parameter is not much effective in this interval. On the other hand, above the horizontal axis wall shear stress $f^{\prime \prime}(0)$ increases for $-2.7<\gamma$ and $g^{\prime \prime}(0)$ boost up for $\gamma>2$.7. It is visualized that increasing relaxation time causes more shear to deform the fluid. The shear stress at the wall increases and asymptotic solution agrees with numerical solution on the upper half of the plot as the value of relaxation time parameter $\beta_{1}$ increases.

Figure 3 exhibit the behavior of displacement thicknesses $\delta_{x} \delta_{y}$ and $\delta_{1}$ over $\gamma$ ranges from $-10 \leq \gamma \leq 10$. The comparison between displacement thicknesses for $\beta_{1}=0.0, \beta_{1}=0.01$ and $\beta_{1}=0.02$ are plotted. The solid lines represent numerical outcomes while dotted lines show asymptotic behavior for large- $\gamma$. It can be seen that like shear stresses, the displacement thicknesses also show reflection symmetry about $\gamma=0$. All thicknesses are equal for axisymmetric case $(\gamma=0)$ with maximum value 0.519 . The displacement thicknesses $\delta_{x}, \delta_{y}$ are maximum at $\gamma= \pm 5$. For large- $\gamma$, thickness is reduced down as relaxation time parameter increases. Similar behavior can be seen for the asymptotic solutions of these profiles. It is clear from the Fig. 3 that for $-4<\gamma$, $\delta_{x}$ decreases remarkably, on the other hand $\delta_{y}$ decreases for $\gamma>4$. The three-dimensional boundary layer thickness $\delta_{1}$ increases for $\gamma \rightarrow \pm \infty$. No remarkable change of these thicknesses seemed near axisymmetric case between the intervals $-5 \leq \gamma \leq 5$. In this particular region, all thicknesses coincide to their respective thickness for a viscous fluid. The asymptotic solution for $\delta_{1}$ move away from the numerical solution for $\beta_{1}$. This means that asymptotic solution moves away from the numerical result as relaxation time boosts up. The boundary layer thickness in $\mathrm{x}$ and $\mathrm{y}$ direction becomes thin as $\gamma$ tends to have positive or negative infinity. These thicknesses become more significant in the axisymmetric case as opposed to non-axisymmetric. Three-dimensional boundary layer thickness becomes thicker for larger value of relaxation time parameter. This implies that the fluids with greater viscoelastic effect causes three-dimensional boundary layer thickness get thicker.
Fig. 3 Variation in displacement thicknesses $\delta_{x}, \delta_{y}$ and $\delta_{1}$ along $\gamma$ for some selected values of $\beta_{1}$. Solid lines represent numerical solutions whereas dotted lines indicate asymptotic behaviors for large- $\gamma$

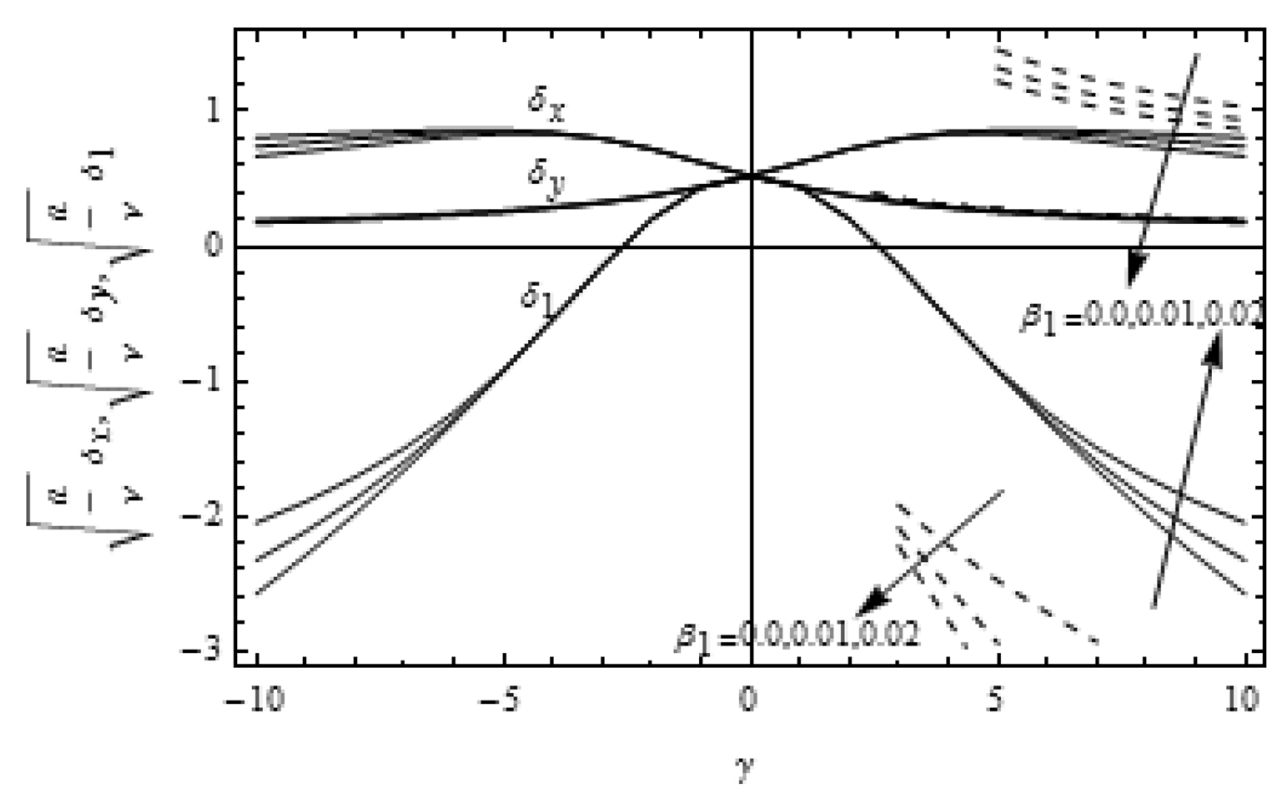


Fig. 4 Similarity velocity profiles $f^{\prime}(\eta)$ for $\beta_{1}=0.03$ at the selected positive values of $\gamma$

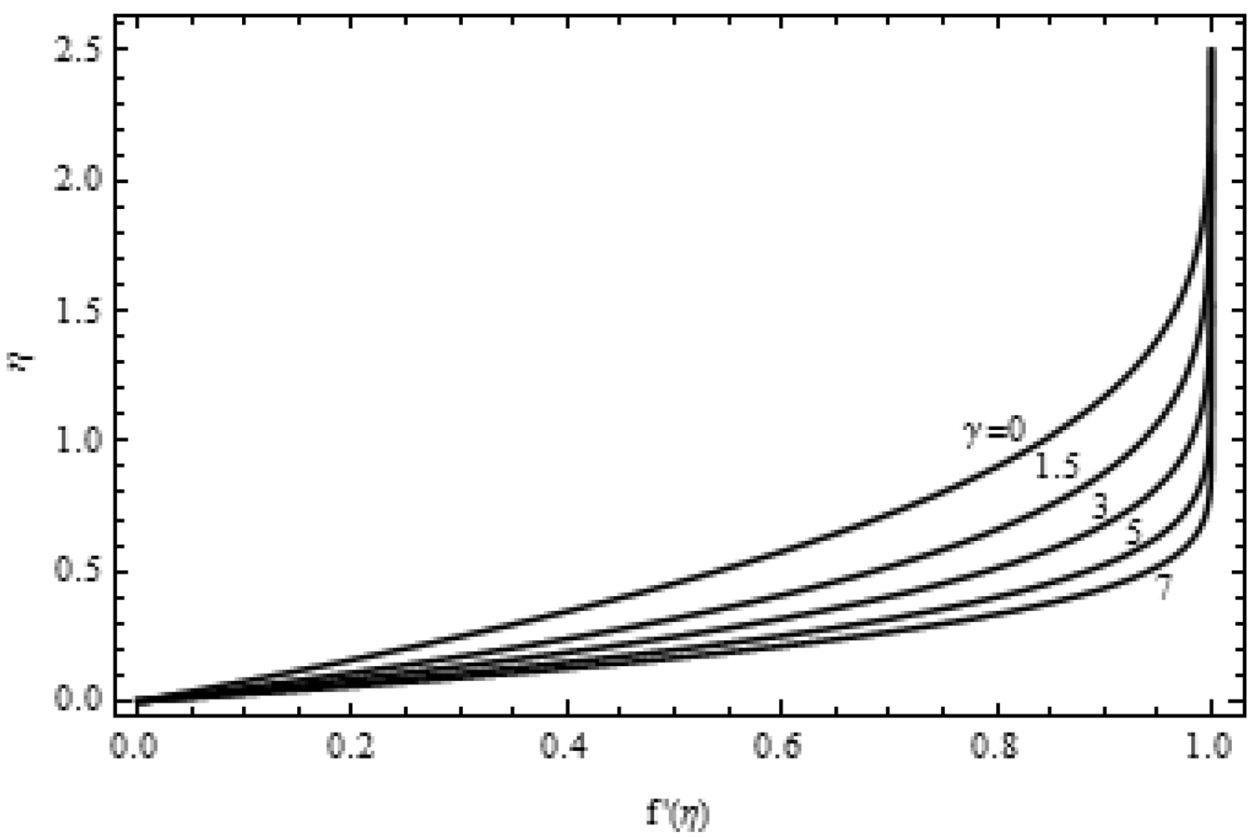

Figure 4 shows the similarity profiles $f^{\prime}(\eta)$ for some selected values of $\gamma$ when $\beta_{1}=0.03$. The Homman axisymmetric behavior of $f^{\prime}(\eta)$ can be seen for $\gamma=0$. With the increase in the values of ratio $\gamma$ the velocity profile $f^{\prime}(\eta)$ falls. Similar behavior can be observed by raising $\beta_{1}$, the velocity profile $g^{\prime}(\eta)$ also reduces. It suggests shear-tostrain rate ratio opposes the velocity of the fluid. The velocity profile for viscous fluid flow toward non-axisymmetric stagnation-point are achieved for $\beta_{1}=0$. Figure 5 represents the similarity profile $g^{\prime}(\eta)$ for some selected values $\gamma$ when $\beta_{1}=0.03$. The similarity profile $g^{\prime}(\eta)$ exhibits axisymmetric Homman profile for $\gamma=0$. By the variation of the values of $\gamma$ from 0 to 7 , it is clear that for different values of $\gamma$, the reverse flow occurs in the inward direction towards the stagnation point near the wall. When $\beta_{1}=0.01$, the reverse flow occurs at $\gamma>2.5$, however, when $\beta_{1}=0.02$, reverse flow in $g^{\prime}(\eta)$ is at $\gamma>2.65$. Similarly, when $\beta_{1}=0.03$, reverse flow occurs at $\gamma>2.8$. It is clear from these plots that with the increase in the values of the relaxation time parameter $\beta_{1}$, the reverse flow decreases gradually. Both the velocities decrease as relaxation time
Fig. 5 Similarity velocity profiles $g^{\prime}(\eta)$ for $\beta_{1}=0.03$ at the selected positive values of $\gamma$

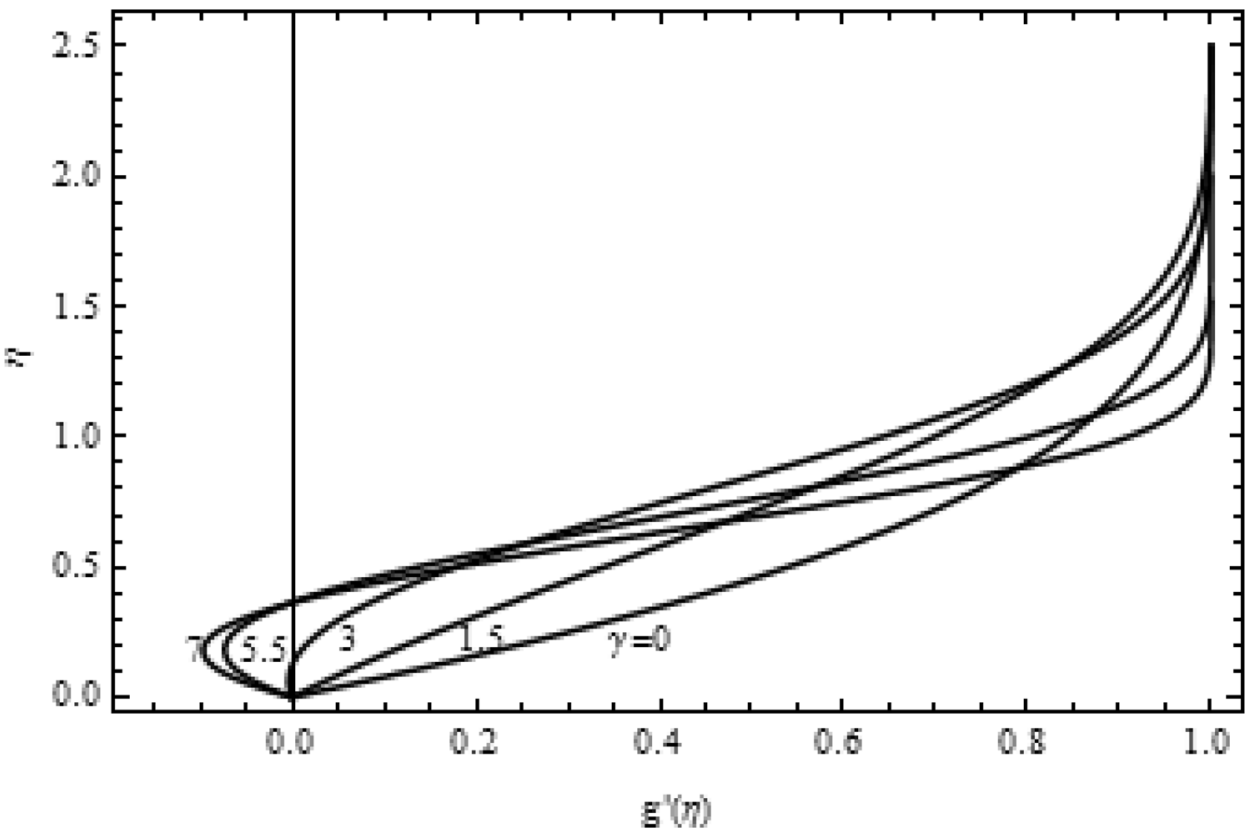

SN Applied Sciences 
Fig. 6 Velocity profiles $f^{\prime}(\eta)$ when $\gamma=5$ at some selected positive values of $\beta_{1}$
Fig. 7 Velocity profiles $g^{\prime}(\eta)$ when $\gamma=5$ at some selected positive values of $\beta_{1}$
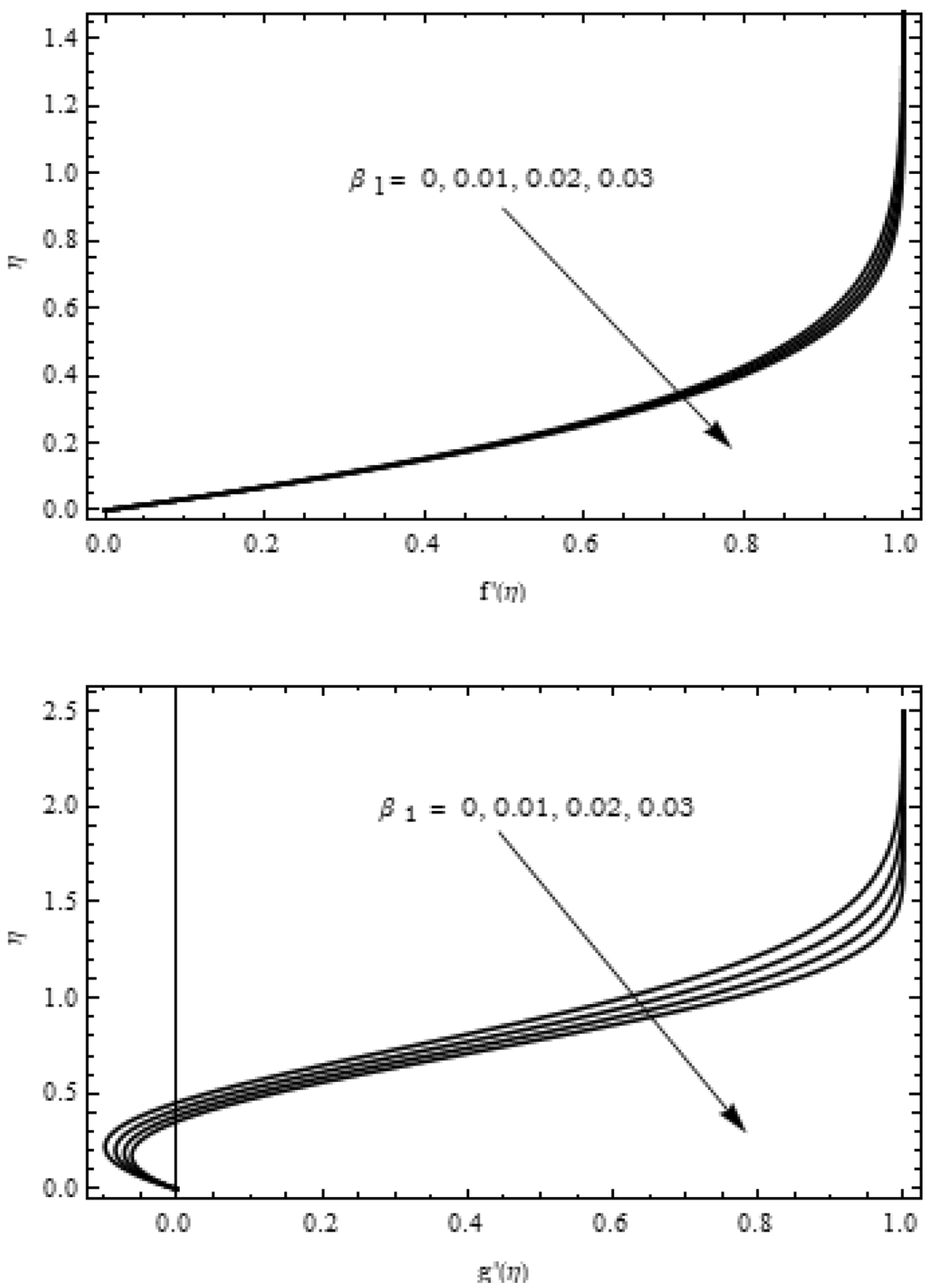

parameter increases. This implies that larger viscous effect results in slowing down the velocity of the fluid.

In Fig. 6 , the velocity profile $f^{\prime}(\eta)$ is presented for $\gamma=5$ when the values of relaxation time parameter $\beta_{1}$ vary. It can be observed that reduction in velocity profile $f^{\prime}(\eta)$ for more the relaxation time $\beta_{1}$. Physically relaxation time parameter $\beta_{1}$ slows down the velocity of the fluid. Similarly, Fig. 7 represents the similarity profile $g^{\prime}(\eta)$ for $\gamma=5$ and selected values of $\beta_{1}$. The effect of the relaxation time parameter $\beta_{1}$ reveals the same behavior as for $f^{\prime}(\eta)$. The velocity of the fluid $g^{\prime}(\eta)$ slows down as the value of $\beta_{1}$ grows larger. The velocity of the fluid in y-direction $g^{\prime}(\eta)$ reduces much faster compared with $f^{\prime}(\eta)$. The reverse flow in $g^{\prime}(\eta)$ also slows down as time relaxation parameter $\beta_{1}$ rises due to viscoelasticity of the fluid. The relaxation time parameter decreases the velocity of the fluid. It affects the reverse flow which can be seen more patterned than viscous fluid. This is due to the fact that Maxwell fluid exhibits more viscoelasticity than viscous fluid as the former needs more shear to deform than the latter. 


\section{Conclusion}

In the present paper, we explored Non-Newtonian fluid towards non-axisymmetric Homann stagnation-point flow. We examined the study of Maxwell fluid for non-axisymmetric stagnation-point by analyzing how Maxwell fluid parameter $\beta_{1}$ affects the velocity profile, wall shear stress parameter, and displacement thicknesses. The perturb solution to these profiles is also established for large $-\gamma$.

It is observed that the wall shear stresses $f^{\prime \prime}(0)$ and $g^{\prime \prime}(0)$ grow gradually in upward direction away from the asymptotic solution for large $-\gamma$ as time relaxation parameter $\beta_{1}$ rises. The displacement thicknesses $\delta_{x}$ and $\delta_{y}$ become thin for Maxwell parameter. Three-dimensional boundary layer thickness $\delta_{1}$ become thicker as relaxation time gets boost up. The similarity profile $f^{\prime}(\eta)$ reduces for the relaxation time parameter $\beta_{1}$. The relaxation time parameter $\beta_{1}$ slows down the velocity profile $g^{\prime}(\eta)$ more rapidly compared with $f^{\prime}(\eta)$. It is observed that the reverse flow occurs for certain value $\gamma(\gamma>2.5)$ in inward direction towards the stagnation point in the vicinity of the wall in $g^{\prime}(\eta)$. The reverse flow reduces when compared with viscous flow as the relaxation time parameter $\beta_{1}$ increases.

\section{Compliance with ethical standards}

Conflict of interest The authors declare that they have no conflict of interest.

Open Access This article is licensed under a Creative Commons Attribution 4.0 International License, which permits use, sharing, adaptation, distribution and reproduction in any medium or format, as long as you give appropriate credit to the original author(s) and the source, provide a link to the Creative Commons licence, and indicate if changes were made. The images or other third party material in this article are included in the article's Creative Commons licence, unless indicated otherwise in a credit line to the material. If material is not included in the article's Creative Commons licence and your intended use is not permitted by statutory regulation or exceeds the permitted use, you will need to obtain permission directly from the copyright holder. To view a copy of this licence, visit http://creativecommons .org/licenses/by/4.0/.

\section{References}

1. Maxwell JC (1867) Iv On the dynamical theory of gases. Phil Tran Royal Soci London 157:49-88

2. Olsson F, Yström J (1993) Some properties of the upper convected Maxwell model for viscoelastic fluid flow. J non-newtonian fluid mech 48:125-145

3. Sadeghy K, Hajibeygi H, Taghavi SM (2006) Stagnation-point flow of upper-convected Maxwell fluids. Int J Non-Linear Mech 41:1242-1247
4. Kumari M, Nath G (2009) Steady mixed convection stagnationpoint flow of upper convected Maxwell fluids with magnetic field. Int J Non-Linear Mech 44:1048-1055

5. Hayat T, Abbas Z, Sajid M (2009) MHD stagnation-point flow of an upper-convected Maxwell fluid over a stretching surface. Chaos Sol Fract 39:840-848

6. Mukhopadhyay S (2012) Heat transfer analysis of the unsteady flow of a Maxwell fluid over a stretching surface in the presence of a heat source/sink. Chin Phy Lett 29:054703

7. Motsa SS, Khan Y, Shateyi S (2012) A new numerical solution of Maxwell fluid over a shrinking sheet in the region of a stagnation point. Math Prob Eng. https://doi.org/10.1155/2012/29061 5

8. Renardy M, Wang X (2012) Boundary layers for the upper convected Maxwell fluid. J Non-Newtonian F Mech 189:14-18

9. Bhattacharyya K, Hayat T, Alsaedi A (2014) Dual solutions in boundary layer flow of Maxwell fluid over a porous shrinking sheet. Chin Phy B 23:124701

10. Ramesh GK (2015) Numerical study of the influence of heat source on stagnation point flow towards a stretching surface of a Jeffrey nanoliquid. J Eng. https://doi.org/10.1155/2015/38206

11. Ramesh GK, Gireesha BJ, Hayat T, Alsaedi A (2016) Stagnation point flow of Maxwell fluid towards a permeable surface in the presence of nanoparticles. Alexandria Eng J 55:857-865

12. Prasannakumara BC, Ramesh GK, Gireesha BJ (2016) Melting and radiation effects on stagnation point Jeffrey fluid flow over a stretching sheet in the presence of nanoparticles. J Nanofluids 5:993-999

13. Mushtaq A, Mustafa M, Hayat T, Alsaedi A (2018) Correction: Buoyancy effects in stagnation-point flow of Maxwell fluid utilizing non-fourier heat flux approach. PLoS One 13:e0200325

14. Kumar KA, Sugunamma V, Sandeep N (2019) Effect of thermal radiation on MHD Casson fluid flow over an exponentially stretching curved sheet. JAnal Calorim 140:2377-2385

15. Tlili I, Nabwey HA, Samrat SP, Sandeep N (2020) 3D MHD nonlinear radiative flow of $\mathrm{CuO}-\mathrm{MgO} /$ methanol hybrid nanofluid beyond an irregular dimension surface with slip effect. Sci Rep 10:1-14

16. Hiemenz K (1911) Die Grenzschicht an einem in den gleichformigen Flussigkeitsstrom eingetauchten geraden Kreiszylinder. Dinglers Pol J 326:321-324

17. Homann F (1936) Der Einfluss grosser Zähigkeit bei der Strömung um den Zylinder und um die Kugel. ZAMM-J App Math Mech 16:153-164

18. Howarth L (1951) CXLIV The boundary layer in three dimensional flow part II. the flow near a stagnation point. Lon Ed Dublin Phil Mag J Sci 42:1433-1440

19. Davey A (1961) Boundary-layer flow at a saddle point of attachment. J Fluid Mech 10:593-610

20. Davey A, Schofield D (1967) Three-dimensional flow near a twodimensional stagnation point. J Fluid Mech 28:149-151

21. Schofield D, Davey A (1967) Dual solutions of the boundary-layer equations at a point of attachment. J Fluid Mech 30:809-811

22. Sajid M, Sadiq MN, Ali N, Javed T (2018) Numerical simulation for Homann flow of a micropolar fluid on a spiraling disk. Eur J Mech-B/Fluids 72:320-327

23. Weidman PD (2012) Non-axisymmetric Homann stagnationpoint flows. J Fluid Mech 702:460-469

24. Mahapatra TR, Sidui S (2017) Heat transfer in non-axisymmetric Homann stagnation-point flows towards a stretching sheet. Eur J Mech-B/Fluids 65:522-529

25. Mahapatra TR, Sidui S (2017) Unsteady heat transfer in nonaxisymmetric Homann stagnation-point flows. Zeitschrift für ang Math Und Phy 68:32

26. Kudenatti RB, Kirsur SR (2017) Numerical and asymptotic study of non-axisymmetric MHD boundary layer stagnation-point flows. Math Meth Appl Sci 40:5841-5850 
27. Lok YY, Merkin JH, Pop I (2017) Mixed convection non-axisymmetric Homann stagnation-point flow. J of Fluid Mech 812:418-434

28. Mahapatra TR, Sidui S (2020) Non-axisymmetric Homann stagnation-point flow of a viscoelastic fluid towards a fixed plate. Eur J Mech-B/Fluids 79:38-43

29. Ahmad I, Jagwal MR, Sajid M (2020) Numerical simulation of non-axisymmetric Homann's stagnation-point flow of nanofluid. J nanofluids 9:47-55

30. Zainal NA, Nazar R, Naganthran K, Pop I (2020) Unsteady threedimensional MHD non-axisymmetric homann stagnation point flow of a hybrid nanofluid with stability analysis. Mathematics 8:784
31. Jagwal MR, Ahmad I, Sajid M (2021) Non-axisymmetric Homann stagnation point flow of Maxwell nanofluid towards fixed surface. Int J Modern Phy C. https://doi.org/10.1142/S012918312 1500765

Publisher's Note Springer Nature remains neutral with regard to jurisdictional claims in published maps and institutional affiliations. 